\title{
A Continuous 3D-Graphene Network to Overcome Threshold Issues and Contact Resistance in Thermally Conductive Graphene Nanocomposites
}

\author{
Federico Conrado and Matteo Pavese \\ Dipartimento di Scienza Applicata e Tecnologia, Politecnico di Torino, Corso Duca degli Abruzzi 24, Torino, Italy \\ Correspondence should be addressed to Federico Conrado; federico.conrado@polito.it
}

Received 5 October 2016; Accepted 4 January 2017; Published 24 January 2017

Academic Editor: Alessandro Pegoretti

Copyright (C) 2017 Federico Conrado and Matteo Pavese. This is an open access article distributed under the Creative Commons Attribution License, which permits unrestricted use, distribution, and reproduction in any medium, provided the original work is properly cited.

\begin{abstract}
In order to overcome thermal resistance issues in polymeric matrix composites, self-standing graphene aerogels were synthetized and infiltrated with an epoxy resin, in order to create conductive preferential pathways through which heat can be easily transported. These continuous highly thermally conductive 3D-structures show, due to the high interconnection degree of graphene flakes, enhanced transport properties. Two kinds of aerogels were investigated, obtained by hydrothermal synthesis (HS) and ice-templated direct freeze synthesis (DFS). Following HS method an isotropic structure is obtained, and following DFS method instead an anisotropic arrangement of graphene flakes results. The density of the structure can be tuned leading to a different amount of graphene inside the final composite. The residual oxygen, known to be detrimental to thermal properties, was removed by thermal treatment before the infiltration process. With 1,25 wt.\% of graphene, using HS method, the thermal conductivity of the polymeric resin was increased by $80 \%$, suggesting that this technique is a valid route to improve the thermal performance of graphene-based composites. When preferential orientation of the filler was present (DFS case), thermal conductivity was increased more than $25 \%$ with a graphene content of only $0,27 \mathrm{wt} . \%$, demonstrating that oriented structures can further improve the thermal transport efficiency.
\end{abstract}

\section{Introduction}

Improving thermal conductivity of materials is a goal of great concern nowadays. There are in fact many applications in which there is the need to remove heat and to dissipate it in the surrounding environment or to recover it in order to improve the efficiency of some process [1].

Generally the materials commonly used nowadays in these fields are metals because of their high values of thermal conductivity that allow the dissipation of a great amount of heat. However sometimes it can be useful to replace these materials with a polymeric based one. The replacement could in fact provide a strong weight reduction of the structure as much as avoiding issues related to corrosion resistance, when there is contact with a highly corrosive medium or when problems related to galvanic coupling creation arise.

Since the polymers are thermal insulators $[1,2]$ there is a strong need to improve their thermal conductivity. To do this, the standard approach consists in dispersing a highly thermally conductive filler inside the polymeric insulating matrix in order to create continuous pathways through which the heat can be transported across the material and then recovered or dissipated $[3,4]$.

Recently much attention has focused on nanofillers such as graphene and nanotubes [5-7] whose outstanding thermal conductivity could provide a high enhancement on the thermal conductivity of the composite. Regarding graphene, however, in order to obtain a performing material, some issues, such as the dimension of the flakes, the continuity of the structure, the contact between the graphene flakes, and the purity of graphene, have to be taken into account. In the literature there are many works related to this topic [8] and it is possible to see that many parameters influence the thermal conductivity outcome. In particular, there are some problems related to the good dispersion and distribution of the filler inside the matrix, in fact generally the better 
the dispersion and distribution the better the final thermal behavior. Ahmadi-Moghadam and Taheri [9] studied the dispersion and distribution of graphene flakes after a roll mill process by SEM morphology observation and thermal conductivity measurements. The results show how the best thermal conductivity was obtained when the bridging distance of the flakes was as big as possible, reaching the highest value of $0,415 \mathrm{Wm}^{-1} \mathrm{~K}^{-1}$ with $2 \%$ of filler (whose initial lateral size was $25 \mu \mathrm{m}$ ). Another key factor is in fact the dimension of the graphene flakes. The work of Fugallo et al. [10] in fact shows that the thermal conductivity of graphene is dependent on the dimension of the flake itself; when its dimension is increased more phonons modes with longer mean free path became available. In addition, the smaller the flake the smaller the thermal conductivity since the thermal resistance grows due to the high number of contacts between the graphene flakes. Many works were made using graphene with small lateral dimensions and the thermal conductivity achievable at low filler loading is really small as proved by the literature $[9,11-15]$. The reason is the quantity and the quality of the contacts between the graphene flakes, since the contacts act as active centers of thermal resistance of the composite if they are not well studied and developed.

In this study we propose a way to increase the efficiency of thermal transport and thus to minimize the thermal resistance inside the polymer thermally insulating matrix. The strategy consists of creating beforehand a highly thermally conductive continuous 3D structure that will be embedded into a polymeric resin to achieve a highly thermally conductive polymeric based nanocomposite. In the literature various techniques can be used to obtain continuous $3 \mathrm{D}$ networks [16, 17]. In particular, regarding the structure of graphene aerogels much work has been done $[18,19]$ and many papers can be found regarding the formation and engineering of aerogels [20-31]. Among all the different syntheses described in the literature that allow the creation of these $3 \mathrm{D}$ structures, the hydrothermal synthesis (HS) and the direct freeze synthesis (DFS) were used in this paper. With these methods it was possible to obtain either isotropic (HS) or anisotropic (DFS) self-standing graphene aerogels.

These highly thermally conducting 3D structures were then infiltrated with an epoxy resin, in order to create polymeric nanocomposites which have, due to the strong interconnection of graphene planes, enhanced transport properties. The porosity of the aerogels can be tuned leading to a different density of the structure and thus to a different amount of filler inside the final composite material.

In the literature 3D structures are created and used for several applications $[22,24,26-28,30-36]$. In particular, papers are often focused on the creation of a continuous structure to improve the electrical conductivity [30, 31, 3335] but also to obtain a high efficient thermal transport [32]. In the field of electrical conductivity the creation of the structure could provide outstanding improvement at very small filler content, since the difference between the electrical conductivity of the filler and the matrix is exceedingly high. In addition, as soon as the continuity is guaranteed, the conductivity of the composite rises sharply. In the case of thermal conductivity instead the improvement provided by the filler is less evident. In fact, thermal transport in graphene-based composites is highly hampered by the presence of defects $[10,37]$. In particular the amount of $\mathrm{sp}^{3}$ carbon in graphene was demonstrated to be most detrimental for thermal conductivity, since thermal transport in graphene is mostly due to phonons. Instead, in the case of electrical conductivity, the effect is less pronounced since the electrons are less affected by the presence of $\mathrm{sp}^{3}$ regions. With the aim of removing the $\mathrm{sp}^{3}$ defects, Shen et al. and Xin et al. [38, 39] proposed thermal annealing, demonstrating that it is a valid route to improve thermal conductivity of graphene. For this reason both HS and DFS structures synthetized in this work underwent a thermal treatment at very high temperature to improve the thermal transport efficiency.

\section{Materials and Methods}

2.1. Materials. Bisphenol-a-diglycidyl ether (BADGE) and hexamethylenediamine $\left(\mathrm{H}_{2} \mathrm{~N}\left(\mathrm{CH}_{2}\right)_{6} \mathrm{NH}_{2}\right.$, hexane-1,6-diamine, HMDA) were chosen as oligomer and curing agent to create the polymeric matrix and were purchased from Sigma Aldrich.

Ultra-highly concentrated graphene oxide (GO) solution $(6.2 \mathrm{mg} / \mathrm{ml}$, flake dimension ranging from 0.5 to $5 \mu \mathrm{m})$ was chosen to create the graphene oxide aerogels and was purchased from Graphene Supermarket.

2.2. Method. Composites were created by polymeric infiltration of 3D self-standing graphene aerogels prepared following two kinds of synthesis: hydrothermal synthesis (HS) and direct freeze synthesis (DFS), followed by freeze drying. The HS method consists of heat treating at $180-230^{\circ} \mathrm{C}$ the GO solution inside an hydrothermal reactor, in order to obtain a carbon spongy isotropic material [20]. During the HS process, the graphene oxide in the solution is partially reduced and its solubility in water decreases; as a consequence agglomeration of the flakes occurs in the solution. The migration of the flakes leads to the formation of a porous aggregate whose volume is considerably lower than the one of the starting solution [18]; even if its solid content is still very low, the relative density is in the few percent range. In the case of HS, as demonstrated in the literature, the densification of the GO structure is completed after a critical time [20]. Therefore, in order to tune the density of the structure, the main parameters to set are the time of HS process and the concentration of the starting GO solution (which determines the quantity of graphene present in the final structures).

The DFS method consists of sharp cooling of the GO solution by liquid nitrogen immersion. In this case the creation of ice dendrites drives the building of graphene walls at the boundaries between the forming crystals, giving rise to a highly oriented structure in the direction of the primary dendrites [29]. In this case the scaffold is selfstanding because of the huge amount of polar bonds created between the graphene oxide flakes.

With DFS the volume of the final structure is the same as the volume of the solution, since there is no densification. For this reason in the case of DFS only the maximum available concentration of the starting graphene oxide solution was 
investigated in order to have a final structure as dense as possible.

After HS and DFS, freeze drying was performed for 24$30 \mathrm{~h}$ in order to sublimate the ice without the collapsing of the structure. In the case of HS, the used samples were cylinders with diameter from 13 to $20 \mathrm{~mm}$ and height from 12 to $20 \mathrm{~mm}$; the density obtained after the freeze drying process ranged between $7 \cdot 10^{-3} \mathrm{~g} / \mathrm{cm}^{3}$ and $17 \cdot 10^{-3} \mathrm{~g} / \mathrm{cm}^{3}$. In the case of DFS the used samples were cylinders with diameter of $20 \mathrm{~mm}$ and height of $10 \mathrm{~mm}$; the density after freeze drying was lower, $5 \cdot 10^{-3} \mathrm{~g} / \mathrm{cm}^{3}$.

Thermal annealing of the structures prior to infiltration was performed in mild vacuum at $1700^{\circ} \mathrm{C}$ to completely reduce graphene oxide to graphene.

2.3. Characterization. Freeze drying was carried out using a three-shelf Christ alpha 2-4 LD equipment with a condenser temperature of $-85^{\circ} \mathrm{C}$ connected to a Vacuubrand $\mathrm{RZ} 6$ vacuum pump $\left(4 \cdot 10^{-4} \mathrm{mbar}\right)$. Each sample was freeze dried for 24-30 h to complete the freeze drying process.

The morphology of the materials was observed by using a field emission electron scanning microscopy FE-SEM Zeiss MERLIN.

The heat treatment on the aerogels was performed at $1700^{\circ} \mathrm{C}$ for $1 \mathrm{~h}$ using a Pro. Ba. vacuum oven under mild vacuum (0.5 mbar).

$\mathrm{X}$-ray photoelectron spectroscopy (XPS) was performed using a PHI 5000 Versaprobe scanning X-ray photoelectron spectrometer (monochromatic $\mathrm{Al} \mathrm{K} \alpha$ X-ray source with $1486.6 \mathrm{eV}$ energy). The semiquantitative atomic compositions and deconvolution procedures were obtained using Multipak 9.6 dedicated software. All core-level peak energies were referenced to $\mathrm{C} 1$ s peak at $284.5 \mathrm{eV}\left(\mathrm{C}-\mathrm{C} / \mathrm{C}-\mathrm{H} \mathrm{sp}^{2}\right.$ bonds $)$ and the background contribution in HR scans was subtracted by means of a Shirley function.

Thermogravimetric analysis (TGA) was carried out in argon using a Mettler-Toledo TGA/SDTA 851e instrument in the temperature range $25-1500^{\circ} \mathrm{C}$ with a heating rate of $10^{\circ} \mathrm{C} / \mathrm{min}$. All curves were normalized to the unit weight of the sample.

Thermal conductivity of materials was evaluated using a hot disk thermal constants analyzer (Hot Disk TPS 2500s); each specimen was tested five times and the average thermal conductivity was reported. Before the measurement, the composites were polished down to a 2000 grit polishing paper and the thermal conductivity was measured by applying the hot disk sensor on the polished surface. The measurements were performed using the instrument single side isotropic mode at room temperature $\left(23^{\circ} \mathrm{C}\right)$.

Electrical conductivity was calculated by using an Agilent 34420A NanoVolt/Micro-Ohm Meter equipment. Resistance was measured at room temperature $\left(23^{\circ} \mathrm{C}\right)$ using a four-point setup, by placing the clips on the polished surfaces without any conductive paste.

\section{Results and Discussions}

In order to create a continuous graphene network, graphene oxide aerogels were prepared by two kinds of synthesis:
TABLE 1: Atomic composition of the structures made following different syntheses (DFS and HS) and heat treatment (TT).

\begin{tabular}{lccc}
\hline \multirow{2}{*}{ Atomic\% } & \multicolumn{3}{c}{ Scaffolds } \\
& DFS & HS & TT \\
\hline Carbon & 67.3 & 87.0 & 99.3 \\
Oxygen & 32.7 & 13.0 & 0.7 \\
\hline
\end{tabular}

hydrothermal synthesis (HS) and direct freeze synthesis (DFS); both methods start from the graphene oxide solution.

After the preparation of the hydrogels, freeze drying was performed in order to remove the water contained into the carbon scaffolds without collapsing the structure, leading to a creation of a carbon-based aerogel. These structures have been studied in the literature [18-29] and they are relatively strong self-standing structures.

The morphology of the obtained structures was observed by FESEM and is reported in Figure 1. As shown, all the structures obtained are highly continuous and interconnected, with sharp edges and thin wall thickness. It is also clear that there is the possibility of tuning the density of the structures by acting on the concentration of the starting graphene oxide solution. The aerogel shown in Figure 1(a), corresponding to a graphene concentration in the solution of $2 \mathrm{~g} / \mathrm{ml}$, is denser than the aerogels shown in Figure $1(\mathrm{~b})(1 \mathrm{~g} / \mathrm{ml})$ and Figure $1(\mathrm{c})(0.5 \mathrm{~g} / \mathrm{ml})$. It is also clear how by following the HS method the final structure is isotropic, whether in the case of DFS the structure is highly oriented in the direction of dendrites growth, thus anisotropic properties are expected.

The oxygen content of the prepared structures was investigated using XPS analysis, whose results are reported in Figure 2 and Table 1. In both HS and DFS the presence of oxygen inside the scaffold is evident, since the synthesis starts from graphene oxide flakes. In the case of DFS, the oxygen content is the highest $(32.7 \%)$ since the graphene oxide did not undergo any heat treatment but the liquid nitrogen immersion that forces together the graphene oxide flakes between the ice dendrites.

In the case of HS instead, XPS analysis shows only $13.0 \%$ of oxygen; the reason is related to the high temperature and pressure of the process. The high energetic oxygencontaining groups on the surface of graphene flakes start to diffuse on the surface [40] and to react together, leading to a partially reduced graphene oxide. Due to this partial reduction, the flakes tend to adhere to each other during their Brownian movement in the solution, forming a threedimensional structure that is much denser than in the case of DFS.

High resolution carbon and oxygen spectra are shown in Figures 2(b) and 2(c). In the case of DFS structures, the presence of both $\mathrm{sp}^{2}$ and $\mathrm{sp}^{3}$ carbon is observed (peak at 284.5 and $285-286 \mathrm{eV}$, resp.), while in the case of HS the peak related to $\mathrm{sp}^{3}$ carbon is strongly reduced with respect to the case of DFS, even if a small shoulder is still observed on the left of the main $\mathrm{sp}^{2}$ peak, due to the remaining oxygen in the structure.

Oxygen has been proved to be highly detrimental for thermal conductivity in graphene, since it acts as a defect, 


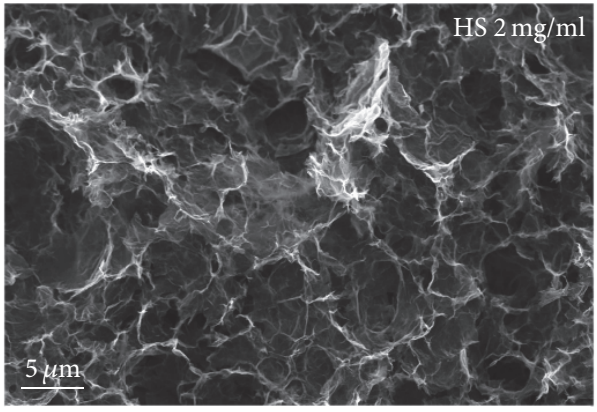

(a)

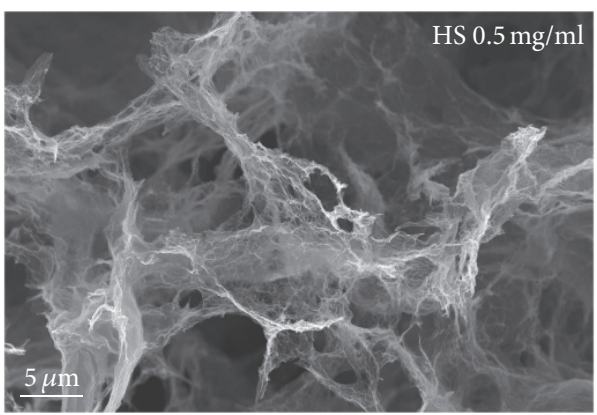

(c)

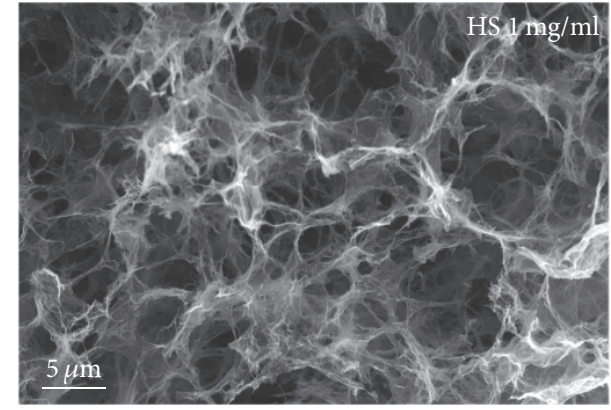

(b)

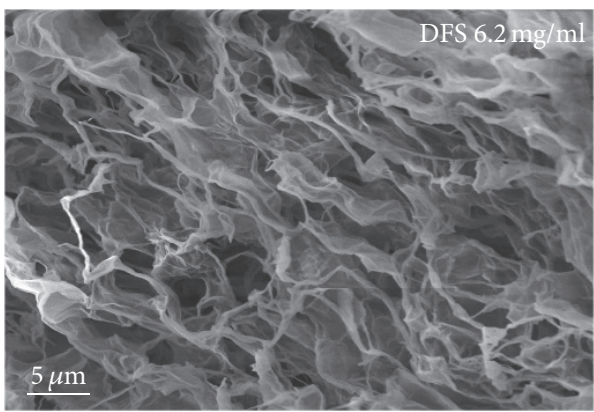

(d)

FIGURE 1: FESEM morphology of graphene oxide aerogels after freeze drying process: (a) $2 \mathrm{mg} / \mathrm{ml}$ GO starting solution, HS method; (b) $1 \mathrm{mg} / \mathrm{ml}$ GO starting solution, HS method; (c) $0.5 \mathrm{mg} / \mathrm{ml} \mathrm{GO}$ starting solution, HS method; (d) $6.2 \mathrm{mg} / \mathrm{ml}$ GO starting solution, DFS method.

breaking the $\mathrm{sp}^{2}$ structure and acting as an active center for phonon scattering leading to undesired thermal resistance inside the material $[10,38,39,41]$. In order to remove the oxygen contained on scaffolds a thermal reduction of both $\mathrm{HS}$ and DFS structures was performed at $1700^{\circ} \mathrm{C}$ under $50 \mathrm{~Pa}$ vacuum. The XPS spectrum of the heat treated scaffold (TT) is shown in Figure 2(c). The curves of treated DFS and HS scaffolds are exact replicas, so that only one is shown. The final oxygen content of this structure was as low as $0.7 \%$, meaning that the structure was fully reduced and reverted to pure graphene. High resolution carbon on the TT scaffold shows the disappearing of the $\mathrm{sp}^{3}$ signals present in the case of HS and DFS scaffolds and the presence of the sole $\mathrm{sp}^{2}$ structure. High resolution oxygen shows an almost flat curve in the region of Ols peak (Figure 2(c)).

High resolution on nitrogen shows in all the structures that there is, as expected, no presence of nitrogen. A quantitative analysis of the composition of the structures is here reported in Table 1 .

To confirm the extent of the reduction of graphene oxide happening in the HS case during the hydrothermal treatment, TGA analyses were also performed under inert atmosphere up to $1500^{\circ} \mathrm{C}$ and the results are reported in Figure 3. A different weight loss can be observed in the cases of DFS and HS, due to their different initial oxygen content.

In the case of DFS there is a huge weight loss related to adsorbed water (more than $10 \mathrm{wt} . \%$ at $140^{\circ} \mathrm{C}$ ) because the higher polarity of the surfaces allows more water adsorption.
At higher temperature a significant reduction of the graphene oxide is observed, between 180 and $300^{\circ} \mathrm{C}$, with more than $30 \mathrm{wt} . \%$ loss. In the case of HS instead, the losses at low temperature are much smaller, being only $8 \mathrm{wt} . \%$ at $300^{\circ} \mathrm{C}$. This difference is clearly due to the fact that HS samples underwent the hydrothermal process where most of the less stable oxygen-containing groups were reduced.

At higher temperature the two curves are rather similar, with a gradual reduction of weight and a flattening of the curves close to $1500^{\circ} \mathrm{C}$. The total weight loss is around $70 \%$ for DFS scaffolds and around $40 \%$ for HS ones. These results confirm the hypothesis that after the heat treatment the graphene oxide is completely reduced to graphene, as shown by the extremely low oxygen content measured by XPS.

FESEM images of the structures after the heat treatment were collected in order to verify if the morphology of the scaffold is still the same as before the heat treatment. In Figure 4 a comparison between a scaffold before and after the heat treatment is reported, showing that no significant changes are present. In particular it is still possible to observe, after heat treatment, high interconnection between graphene flakes, thin wall thickness, and sharp edges.

The graphene aerogels were infiltrated after thermal reduction with a stoichiometric liquid mixture of BADGE and HMDA. The infiltration was performed by applying vacuum to the system to let the resin deeply infiltrate the structure and let air bubbles flow out, in order to obtain a fully dense material without significant residual porosity. The 


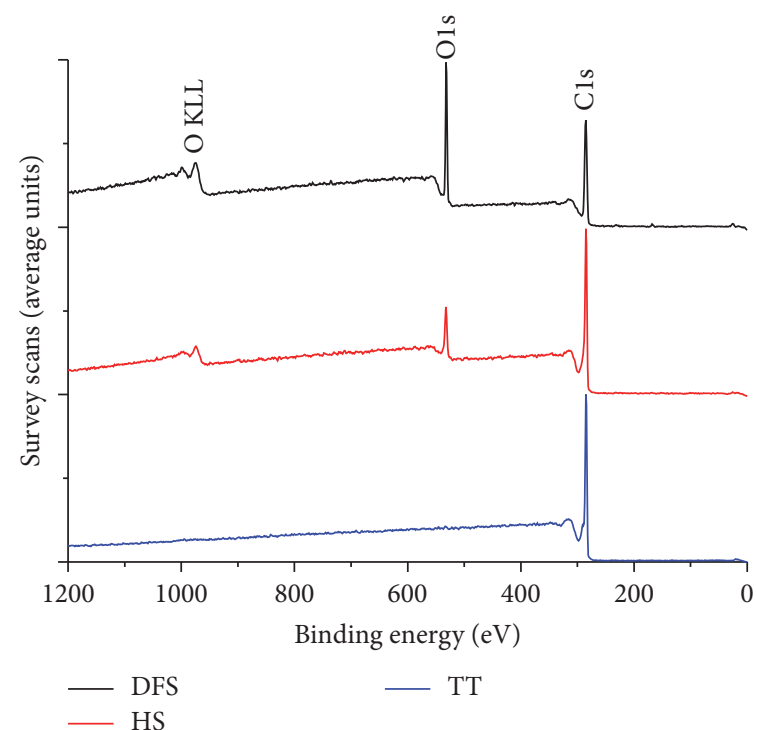

(a)

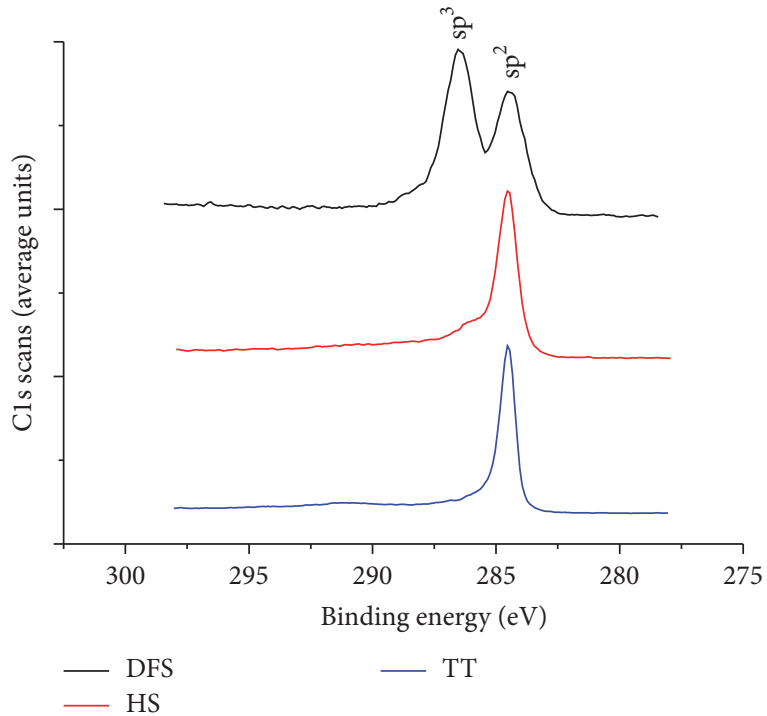

(b)

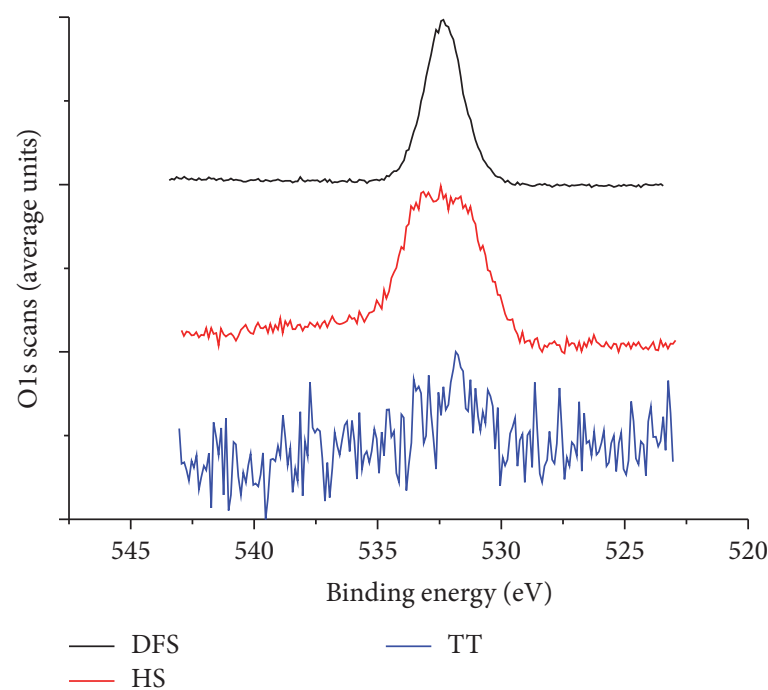

(c)

FIGURE 2: XPS spectra of DFS, HS, and heat treated (TT) scaffolds: (a) survey scans, (b) normalized high resolution scans on Cls, and (c) normalized high resolution scans on O1s.

porosity measured on polished surfaces was always lower than $2 \%$, and the density of both resin and composites was always $1.14 \pm 0.03 \mathrm{~g} / \mathrm{cm}^{3}$.

In order to confirm if the infiltration occurred both microscopy and XPS were used. From optical microscopy and SEM no significant porosity was observed in the bulk of the infiltrated aerogels, suggesting a complete infiltration. To confirm this result, XPS was performed on an internal slice of the composite, and the results are reported in Figure 5. The successful infiltration was confirmed by the presence of nitrogen in all infiltrated samples (Figure 5(a)), since nitrogen is present only in the HMDA curing agent, while it is completely absent in graphene oxide, partially reduced graphene (after HS treatment) and fully reduced graphene (after high temperature treatment). Thus, the presence of the $\mathrm{N}$ peak at $399 \mathrm{eV}$ proves that the polymer completely infiltrated the aerogel. To further verify that this result was not due to artifacts from contamination or polishing, XPS spectra were performed on fracture surface of the materials and at different depths under the surface (between 0.1 and $1 \mathrm{~mm}$ ). The results, shown in Figures 5(b) and 5(c), demonstrate that nitrogen was not due to contamination but that the infiltration was instead complete.

The morphology of the composites was observed by FESEM and is reported in Figure 6 for both the HS and the HS TT infiltrated scaffolds. Both scaffolds are fully infiltrated by the polymer, and some graphene flakes can be seen in both samples. 


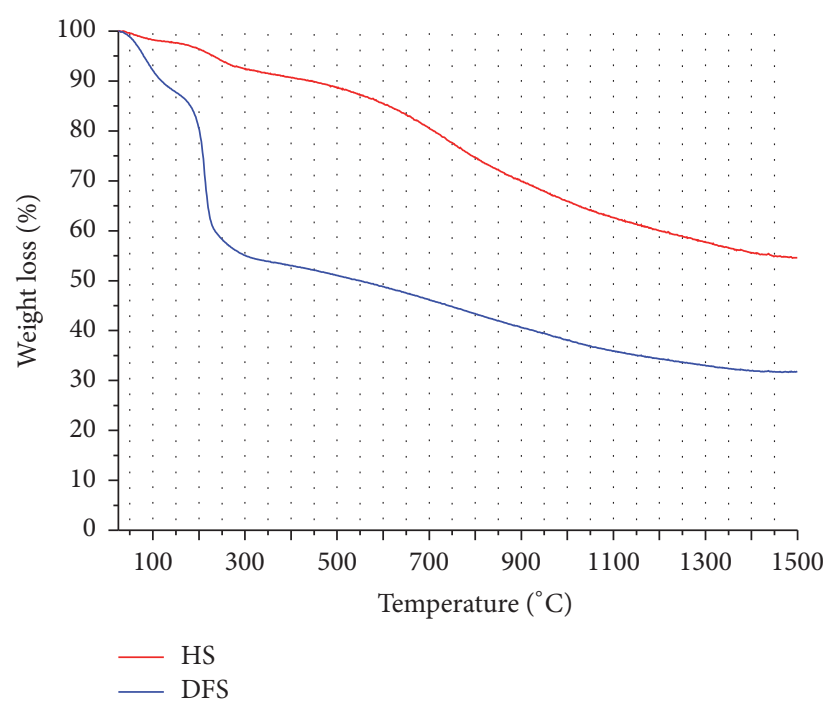

FIGURE 3: TGA analysis of freeze dried scaffolds created by HS and by DFS.

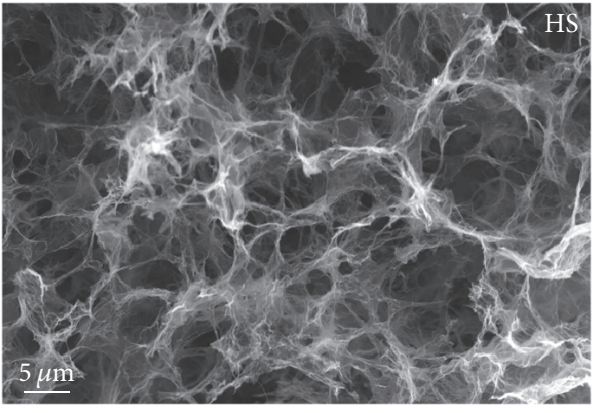

(a)

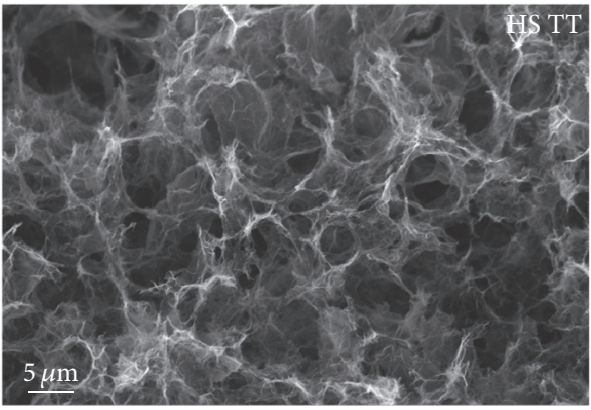

(b)

FIGURE 4: FESEM morphologies of scaffold: (a) before heat treatment and (b) after heat treatment at $1700^{\circ} \mathrm{C}$ under vacuum inert atmosphere.

Thermal conductivity measurements of the composites were carried out using a hot disk analyzer on slices of the composite materials after the infiltration and the curing of the polymer were completed. The results are reported in Figure 7.

The thermal conductivity of the samples obtained by the HS method increases considerably with the graphene content in the composite, reaching an $80 \%$ improvement when $1.25 \mathrm{wt}$.\% of graphene is present. This result is possible due to the high interconnection of the flakes that provides a limited thermal resistance inside the composite material. The 3D network approach in fact solves many issues that are generally observed with standard dispersion approaches, such as the breaking of the continuity of the highly thermally conductive filler by the polymeric insulating matrix, a nonperfect distribution of the filler inside the matrix and a nonperfect contact between the fillers. In this case the conductive pathways through which the heat is transmitted are built before the creation of the composite, thus providing a high distribution of the filler and continuity of the structure. However, in the case of samples obtained by HS method, even if a 3D structure is formed, there is still some problem related to the contact between the flakes. Even if the structure undergoes a heat treatment that provides the graphitization of the flakes, their joining is not optimized yet, mainly because of their orientation. This fact is clearly visible when analyzing the results of samples obtained with the DFS method. The orientation of the structure achievable using this approach can provide a further huge reduction of thermal resistance, as it is evident in Figure 7. With a very low amount of graphene, $0.27 \mathrm{wt} . \%$, a thermal conductivity increase of $25 \%$ with respect to pure polymer can be observed.

The literature on carbon-based nanocomposites for improving thermal properties is rather vast. To compare the literature with the results of this paper, it is first reasonable to consider works where graphene flakes of similar size are used and no 3D structure is built. In this case, as expected, a limited increase in thermal conductivity is observed [11-15]. For instance Prolongo et al. [13] obtained only $6 \%$ thermal conductivity improvement with $0,5 \mathrm{wt} . \%$ of graphene; Chandrasekaran et al. [15] obtained only $6 \%$ and $14 \%$ thermal conductivity improvement with, respectively, 1 and 2 wt.\% of graphene; Ganguli et al. [11] obtained 55\% of thermal conductivity increase with 2 wt.\% of filler. All these works, similarly to the present one, used commercially 


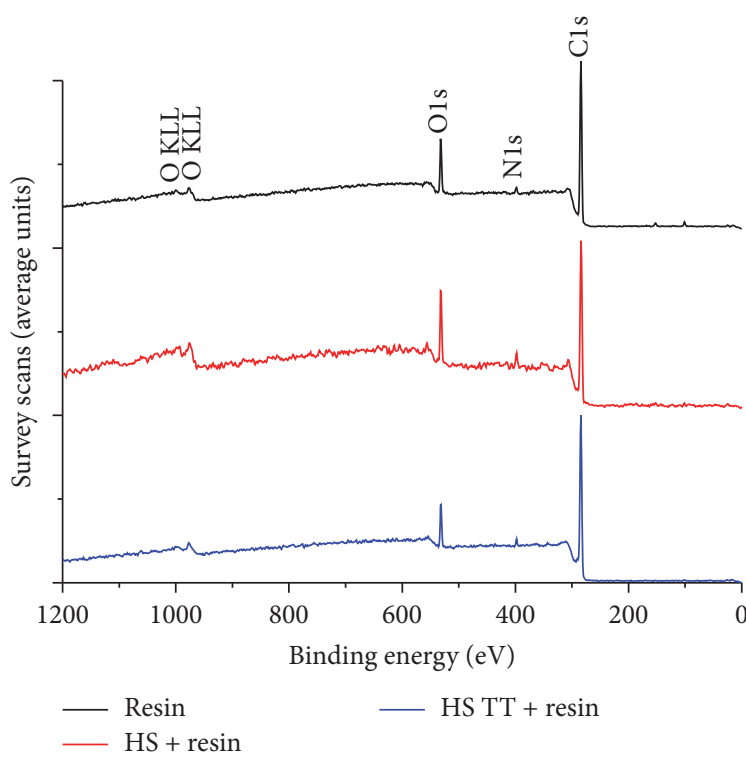

(a)

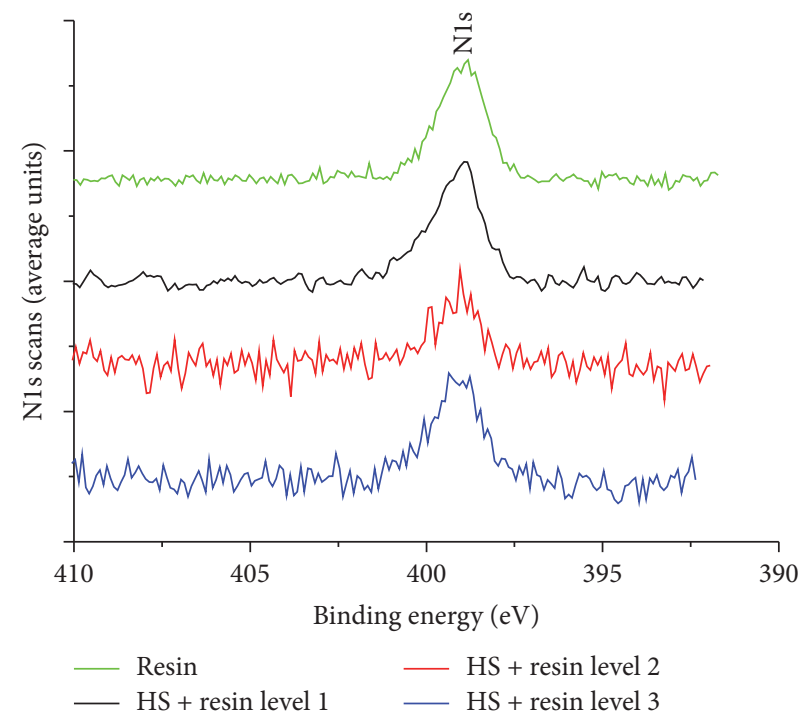

(b)

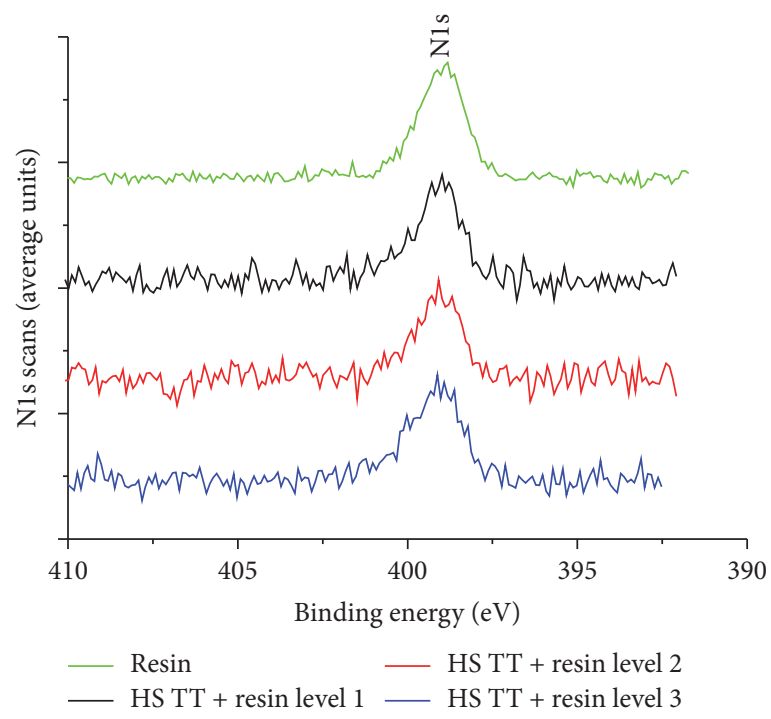

(c)

FIGURE 5: XPS spectra of the composites after the infiltration: (a) survey scan of resin and the composites; (b) high resolution nitrogen on resin and HS scaffold infiltrated with the resin at different depth; (c) high resolution nitrogen on resin and HS TT scaffold infiltrated with the resin at different depth.

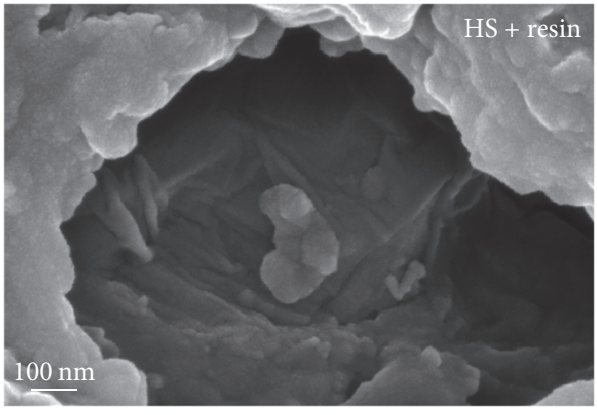

(a)

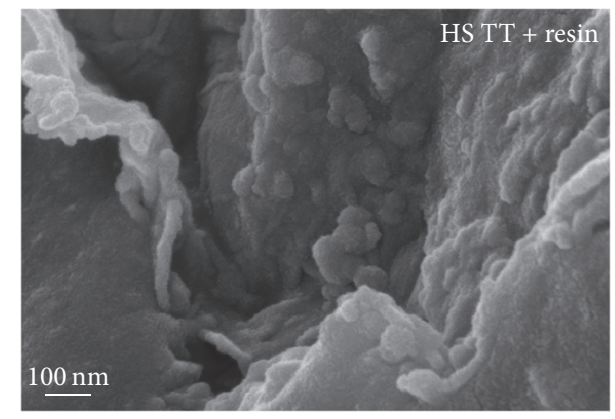

(b)

FIGURE 6: FESEM morphologies of (a) non-heat treated infiltrated scaffold and (b) heat treated infiltrated scaffold. 


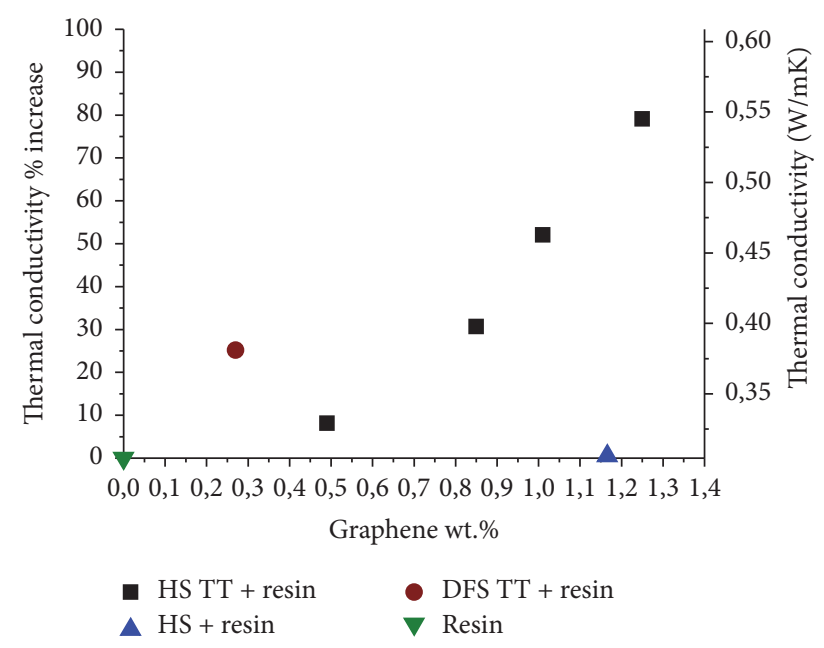

FIgURE 7: Thermal conductivity increases with respect to the thermal conductivity of the pure resin at different carbon contents and for different synthesis methods.

available graphene. There is also some literature works on composites obtaining by dispersing high quality graphene or nanotubes. In this case the purity of the filler, the larger size (in the case of graphene) and the possibility of tuning the process can provide a higher enhancement on the thermal conductivity. For instance Yavari et al. [42] obtained a significant improvement on the thermal conductivity, $70 \%$ and $140 \%$ with 2 and 4 wt.\%, respectively, but to obtain a similar value to HS they use a higher amount of graphene. Similarly Song et al. [43] obtained $1.53 \mathrm{~W} /(\mathrm{m} \mathrm{K})$ with a very high amount of graphene (10 wt.\%) or the smaller value of $0.6 \mathrm{~W} / \mathrm{m} \mathrm{K}$ when using $4 \mathrm{wt} . \%$ of filler. Also in this case the quantity of graphene necessary to obtain a significant improvement of thermal conductivity is rather high. Other researchers use well-dispersed nanotubes to improve thermal properties. For instance Song and Youn [44] provide a $117 \%$ increase of thermal conductivity but a rather low absolute value $(0.26 \mathrm{~W} /(\mathrm{m} \mathrm{K}))$ with $1.5 \mathrm{wt} . \%$ of well-dispersed nanotubes, while Biercuk et al. [45] show a $125 \%$ increase with $1 \%$ of single wall carbon nanotubes. In this case the low percolation threshold of carbon nanotubes allows the formation of a sort of $3 \mathrm{D}$ structure inside the composite that improved thermal conductivity. In fact, it seems that high quality materials and/or high filler fractions are needed to substantially improve the thermal properties of a polymeric material when dispersion techniques are used.

In the literature there can be found only a few works on composites with graphene 3D structures, and among these the best thermal results are described in the paper of Yang et al. [32]. The authors used a mixture of graphene oxide and graphene at different concentration to create a self-standing structure via a "sol-cryo" process, which is then vacuum infiltrated with PEG resin. To evaluate the thermal transport efficiency they define the parameter $\eta=$ $k_{c} /\left(k_{m} \varphi_{G}\right)$, where $\varphi_{G}$ is the mass fraction of filler and $k_{c}$ and $k_{m}$ are the thermal conductivity of the composite and the pure matrix, respectively. Yang et al. reached the remarkable thermal transport efficiency value of 2.6 using at the same time $0.447 \mathrm{wt} . \%$ of GO and $1.786 \mathrm{wt} . \%$ of graphene.

In this work, in the case of HS TT composites the value of thermal transport efficiency is close to 1.5 , which is rather low in comparison to the one obtained by Yang et al. This happens probably due to the fact that the thermal resistance of contacts between the graphene flakes is still high in the case of samples obtained by HS. However, in the case of DFS TT composites, due to the high orientation of flakes, the thermal resistance is much lower, reaching the outstanding value of 4.6, far above the one obtained by Yang et al. This suggests that the proposed approach is a valid route to obtain polymeric nanocomposites with high thermal conducting efficiency.

These results are even more interesting considering that the starting graphene oxide flakes dimensions ranged between 0.5 and $5 \mu \mathrm{m}$. In both HS and DFS syntheses it is expected that increasing the starting dimension of the GO flakes used to create the 3D structure will allow further enhancement on the thermal transport efficiency.

The continuity of the structure provides in addition a great enhancement of the electrical conductivity which is reported in Figure 8.

The electrical conductivity of the composites is increased of around 9 orders of magnitude with respect to the resin, changing the behavior from insulating to electrically conductive for all the composites prepared by using heat treated scaffolds. In particular, it is possible to observe that all the composites, obtained either by the HS or by the DFS methods, after heat treatment, present values between 30 and $500 \mathrm{~S} / \mathrm{m}$. This is an interesting result because in the case of conventional dispersion of commercially available graphene it is generally unusual to increase electrical conductivity with such a low amount of filler; generally to have an electrically conductive polymer a larger amount of filler has to be inserted and dispersed in order to reach the percolation threshold. In fact, as reported by Ahmadi-Moghadam and Taheri [9], in the case of a filler with an average dimension of the graphene flakes below $5 \mu \mathrm{m}$, the percolation is generally achieved only when more than 4 wt.\% is used. Instead, by using this approach there is the possibility of reaching percolation and electrical conduction for values as low as $0.5 \mathrm{wt}$.\%. This fact is further evidenced in the case of DFS method in which there is a high value of electrical conductivity even if the amount of carbon is as low as $0.27 \mathrm{wt} . \%$.

A rather high electrical conductivity value, $0.66 \mathrm{~S} / \mathrm{m}$, was observed also for the composite obtained by infiltrating the non-heat treated scaffold. To explain this unexpected result, it is interesting to cite the work of Kumar and coworkers [40], who demonstrated that graphene annealed at low temperature can have a mixed graphene/graphene oxide microstructure. Indeed, during the annealing process the migration of oxidized polar groups forms regions on the surface of graphene where there is a high density of polar groups and regions where their density is low. Thus there are "oxidized island" and "reduced islands" on the surface of graphene scaffolds. Electrons can cross the material through these conductive paths much more easily than phonons. Since the HS composites are obtained by a hydrothermal treatment at relatively low temperature, it is possible that they behave 


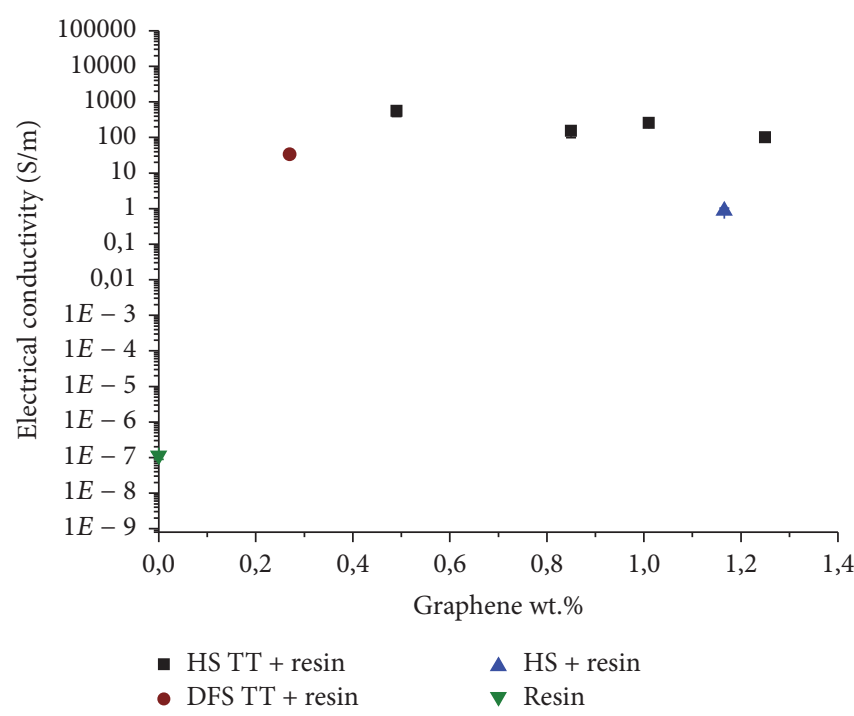

FIGURE 8: Electrical conductivity of the resin and of the composites prepared by resin infiltration of HS TT scaffold (heat treated), DFS TT scaffold (heat treated), and HS (HS scaffold non-heat treated). (The value of the pure resin was out of the range of the instrument so the real value was not detectable because it was below $1.510^{-7} \mathrm{~S} / \mathrm{m}$.)

according to the process proposed by Kumar et al. Thus, as shown in Figure 8, the electrical conductivity is rather high while the thermal conductivity remains low.

The electrical conductivity of the pure resin is reported here only as a calculated value since it was lower than the sensitivity of the instrument (the measured resistance was more than $2 \mathrm{G} \Omega$, instrumental limit, so the electrical conductivity of the resin was less than $1.510^{-7} \mathrm{~S} / \mathrm{m}$ ).

In the literature other authors use the hydrothermal process as a strategy to create self-standing graphene-based structures for improving electrical properties. Their results are fairly comparable with this paper. For example Zhang et al. [30] performed thermal annealing at $800^{\circ} \mathrm{C}$ obtaining $100 \mathrm{~S} / \mathrm{m}$ for a composite with $1 \%$ wt of graphene. Wang et al. [33] reduced the graphene oxide structures using an acidic attack at low temperature $\left(120^{\circ} \mathrm{C}\right)$ during the autoclave process obtaining $20 \mathrm{~S} / \mathrm{m}$. Liu et al. [31] used a mixed graphene-nanotubes structure annealed at $1050^{\circ} \mathrm{C}$ obtaining $16 \mathrm{~S} / \mathrm{m}$, suggesting that all the proposed strategies are valid routes to obtain an electrically conductive material.

Other authors proposed a CVD approach to create a continuous graphene structure on a supporting porous material. For instance, Garlof et al. [34] use a $\mathrm{ZnO}$ scaffold as a template which is then removed during the process, leading to the obtainment of $13.6 \mathrm{~S} / \mathrm{m}$ of electrical conductivity. Jia et al. [35] instead tune the process parameters in order to deposit an average 4-layer graphene coating on a $\mathrm{Ni}$ foam, reaching the outstanding value of $300 \mathrm{~S} / \mathrm{m}$, proving that the CVD approach is a valid route to enhance the electrical conductivity of polymeric based materials despite the higher complexity of the process.

\section{Conclusions}

In the field of thermal conductivity of polymeric based nanocomposites there are many issues to overcome. One of the main difficulties is the high thermal resistance inside the composite that is mostly due to the lack of continuity of the conductive structure and to the imperfect contacts between the highly thermally conductive fillers. In order to overcome these problems there is the need to improve the dispersion and the distribution of the filler inside the matrix, in order to connect the fillers as much as possible, and engineer the contacts between the fillers to reduce the overall thermal resistance. All these objectives can be achieved using the approach proposed in this paper, leading to an interconnected, highly conductive structure that will drive the heat across the material. With the described approach it is possible to obtain both isotropic (HS method) and anisotropic (DFS method) structures with different densities and thus different amounts of graphene in the final composite.

In the case of the DFS it is possible to see how the alignment of the interfaces between the flakes will lead to a better thermal behavior of the composite reaching the $25 \%$ of increase of thermal conductivity with only $0.27 \mathrm{wt} . \%$, an improvement not achievable in the case of HS at this low amount of carbon.

On the other hand using HS $80 \%$ of improvement on the resin thermal conductivity can be achieved with 1.25 wt.\% of filler. This value is hardly obtainable using conventional dispersion methods of commercially available graphene. These results are interesting also because the average lateral size of the starting graphene flakes was small (less than $5 \mu \mathrm{m}$ in the plane), and the higher the lateral surface of the flake the higher the final achievable thermal conductivity improvement.

Also electrical conductivity is greatly improved using this approach, even at very low values of filler content. This is due to the formation of conductive continuous paths before the composite preparation. Due to self-organization of annealed graphene oxide, in the case of HS method, conduction was achieved even without the heat treatment that is used to 
remove oxygen. The improvement after thermal treatment is however much higher, leading to substantially improving the electrical conductivity even when the graphene is present in very low amount.

Graphene foams have been thoroughly studied in the literature [46] and their outstanding properties have been proved in a wide range of applications ranging from electronics devices fabrication to biomedical applications; in this paper, we prove that they are really promising also in the field of thermal conductivity improvement. The scaffolding technique can be a good way to maximize the efficiency of both heat transmission and electrical conductivity inside the composite, minimizing the amount of filler to be inserted, dispersed, and distributed to achieve the desired results.

\section{Competing Interests}

The authors declare that there is no conflict of interest regarding the publication of this paper.

\section{Acknowledgments}

The authors would really like to acknowledge Professor Marco Sangermano who supported and allowed the partnership between Canadian McGill University and Politecnico di Torino that built the foundation of this work, Professor Marta Cerruti for the partnership between McGill and Politecnico di Torino, Professor Paola Palmero for freeze drying equipment, and Professor Renato Gonnelli for the electrical conductivity equipment. The authors acknowledge the support from a Marie Curie International Research Staff Exchange Scheme Fellowship (PHOTOMAT, Proposal no. 318899) within the 7th European Community Framework for funding the research.

\section{References}

[1] C. T. Joen, Y. Park, Q. Wang, A. Sommers, X. Han, and A. Jacobi, "A review on polymer heat exchangers for HVAC \& R applications," International Journal of Refrigeration, vol. 32, no. 5, pp. 763-779, 2009.

[2] Y. Agari, A. Ueda, Y. Omura, and S. Nagai, “Thermal diffusivity and conductivity of PMMA/PC blends," Polymer, vol. 38, no. 4, pp. 801-807, 1997.

[3] J. Hong, D. Park, and S. Shim, "A review on thermal conductivity of polymer composites using carbon-based fillers : carbon nanotubes and carbon fibers," Carbon letters, vol. 11, no. 4, pp. 347-356, 2010.

[4] D. M. Bigg, "Thermal conductivity of heterophase polymer compositions," in Thermal and Electrical Conductivity of Polymer Materials, vol. 119 of Advances in Polymer Science, pp. 1-30, Springer, Berlin, Germany, 1995.

[5] C. Lee, X. Wei, J. W. Kysar, and J. Hone, "Measurement of the elastic properties and intrinsic strength of monolayer graphene," Science, vol. 321, no. 5887, pp. 385-388, 2008.

[6] A. A. Balandin, S. Ghosh, W. Bao et al., "Superior thermal conductivity of single-layer graphene," Nano Letters, vol. 8, no. 3, pp. 902-907, 2008.

[7] P. S. Goh, A. F. Ismail, and B. C. Ng, "Directional alignment of carbon nanotubes in polymer matrices: contemporary approaches and future advances," Composites Part A: Applied Science and Manufacturing, vol. 56, pp. 103-126, 2014.

[8] V. Singh, D. Joung, L. Zhai, S. Das, S. I. Khondaker, and S. Seal, "Graphene based materials: past, present and future," Progress in Materials Science, vol. 56, no. 8, pp. 1178-1271, 2011.

[9] B. Ahmadi-Moghadam and F. Taheri, "Effect of processing parameters on the structure and multi-functional performance of epoxy/GNP-nanocomposites," Journal of Materials Science, vol. 49, no. 18, pp. 6180-6190, 2014.

[10] G. Fugallo, A. Cepellotti, L. Paulatto, M. Lazzeri, N. Marzari, and F. Mauri, "Thermal conductivity of graphene and graphite: collective excitations and mean free paths," Nano Letters, vol. 14, no. 11, pp. 6109-6114, 2014.

[11] S. Ganguli, A. K. Roy, and D. P. Anderson, "Improved thermal conductivity for chemically functionalized exfoliated graphite/epoxy composites," Carbon, vol. 46, no. 5, pp. 806-817, 2008.

[12] F. Wang, L. T. Drzal, Y. Qin, and Z. Huang, "Mechanical properties and thermal conductivity of graphene nanoplatelet/epoxy composites," Journal of Materials Science, vol. 50, no. 3, pp. 10821093, 2016.

[13] S. G. Prolongo, R. Moriche, A. Jiménez-Suárez, M. Sánchez, and A. Ureña, "Epoxy adhesives modified with graphene for thermal interface materials," Journal of Adhesion, vol. 90, no. 10, pp. 835847, 2014.

[14] F.-P. Du, W. Yang, F. Zhang et al., "Enhancing the heat transfer efficiency in graphene-epoxy nanocomposites using a magnesium oxide-graphene hybrid structure," ACS Applied Materials and Interfaces, vol. 7, no. 26, pp. 14397-14403, 2015.

[15] S. Chandrasekaran, C. Seidel, and K. Schulte, "Preparation and characterization of graphite nano-platelet (GNP)/epoxy nano-composite: mechanical, electrical and thermal properties," European Polymer Journal, vol. 49, no. 12, pp. 3878-3888, 2013.

[16] Y. Ma and Y. Chen, "Three-dimensional graphene networks: synthesis, properties and applications," National Science Review, vol. 2, no. 1, pp. 40-53, 2015.

[17] Y.-H. Zhao, Z.-K. Wu, and S.-L. Bai, "Study on thermal properties of graphene foam/graphene sheets filled polymer composites," Composites Part A: Applied Science and Manufacturing, vol. 72, pp. 200-206, 2015.

[18] V. Chabot, D. Higgins, A. Yu, X. Xiao, Z. Chen, and J. Zhang, "A review of graphene and graphene oxide sponge: material synthesis and applications to energy and the environment," Energy and Environmental Science, vol. 7, no. 5, pp. 1564-1596, 2014.

[19] M. Zeng, W. Wang, and X. Bai, "Preparing three-dimensional graphene architectures: review of recent developments," Chinese Physics B, vol. 22, no. 9, 2013.

[20] Y. Xu, K. Sheng, C. Li, and G. Shi, "Self-assembled graphene hydrogel via a one-step hydrothermal process," ACS Nano, vol. 4, no. 7, pp. 4324-4330, 2010.

[21] H. Bi, K. Yin, X. Xie et al., "Low temperature casting of graphene with high compressive strength," Advanced Materials, vol. 24, no. 37, pp. 5124-5129, 2012.

[22] L. Zhang and G. Shi, "Preparation of highly conductive graphene hydrogels for fabricating supercapacitors with high rate capability," Journal of Physical Chemistry C, vol. 115, no. 34, pp. 17206-17212, 2011. 
[23] Z. Han, Z. Tang, P. Li, G. Yang, Q. Zheng, and J. Yang, "Ammonia solution strengthened three-dimensional macroporous graphene aerogel," Nanoscale, vol. 5, no. 12, pp. 54625467, 2013.

[24] P. Chen, J.-J. Yang, S.-S. Li et al., "Hydrothermal synthesis of macroscopic nitrogen-doped graphene hydrogels for ultrafast supercapacitor," Nano Energy, vol. 2, no. 2, pp. 249-256, 2013.

[25] Y. Zhao, C. Hu, Y. Hu, H. Cheng, G. Shi, and L. Qu, "A versatile, ultralight, nitrogen-doped graphene framework," Angewandte Chemie-International Edition, vol. 124, no. 45, pp. 11533-11537, 2012.

[26] Y. Zhao, J. Liu, Y. Hu et al., "Highly compression-tolerant supercapacitor based on polypyrrole-mediated graphene foam electrodes," Advanced Materials, vol. 25, no. 4, pp. 591-595, 2013.

[27] Z.-S. Wu, A. Winter, L. Chen et al., "Three-dimensional nitrogen and boron co-doped graphene for high-performance allsolid-state supercapacitors," Advanced Materials, vol. 24, no. 37, pp. 5130-5135, 2012.

[28] J. Zhao, W. Ren, and H.-M. Cheng, "Graphene sponge for efficient and repeatable adsorption and desorption of water contaminations," Journal of Materials Chemistry, vol. 22, no. 38, pp. 20197-20202, 2012.

[29] J. Kuang, L. Liu, Y. Gao et al., "A hierarchically structured graphene foam and its potential as a large-scale strain-gauge sensor," Nanoscale, vol. 5, no. 24, pp. 12171-12177, 2013.

[30] Q. Zhang, X. Xu, H. Li, G. Xiong, H. Hu, and T. S. Fisher, "Mechanically robust honeycomb graphene aerogel multifunctional polymer composites," Carbon, vol. 93, pp. 659-670, 2015.

[31] X. Liu, H. Li, Q. Zeng et al., "Electro-active shape memory composites enhanced by flexible carbon nanotube/graphene aerogels," J. Mater. Chem. A, vol. 3, no. 21, pp. 11641-11649, 2015.

[32] J. Yang, G.-Q. Qi, Y. Liu et al., "Hybrid graphene aerogels/phase change material composites: thermal conductivity, shapestabilization and light-to-thermal energy storage," Carbon, vol. 100, pp. 693-702, 2016.

[33] Z. Wang, X. Shen, M. Akbari Garakani et al., "Graphene aerogel/epoxy composites with exceptional anisotropic structure and properties," ACS Applied Materials and Interfaces, vol. 7, no. 9, pp. 5538-5549, 2015.

[34] S. Garlof, T. Fukuda, M. Mecklenburg et al., "Electromechanical piezoresistive properties of three dimensionally interconnected carbon aerogel (Aerographite)-epoxy composites," Composites Science and Technology, vol. 134, pp. 226-233, 2016.

[35] J. Jia, X. Sun, X. Lin, X. Shen, Y.-W. Mai, and J.-K. Kim, "Exceptional electrical conductivity and fracture resistance of $3 \mathrm{D}$ interconnected graphene foam/epoxy composites," ACS Nano, vol. 8, no. 6, pp. 5774-5783, 2014.

[36] F. Irin, S. Das, F. O. Atore, and M. J. Green, "Ultralow percolation threshold in aerogel and cryogel templated composites," Langmuir, vol. 29, no. 36, pp. 11449-11456, 2013.

[37] M. Tortello, S. Colonna, M. Bernal et al., "Effect of thermal annealing on the heat transfer properties of reduced graphite oxide flakes: a nanoscale characterization via scanning thermal microscopy," Carbon, vol. 109, pp. 390-401, 2016.

[38] B. Shen, W. Zhai, and W. Zheng, "Ultrathin flexible graphene film: an excellent thermal conducting material with efficient EMI shielding," Advanced Functional Materials, vol. 24, no. 28, pp. 4542-4548, 2014.

[39] G. Xin, H. Sun, T. Hu et al., "Large-area freestanding graphene paper for superior thermal management," Advanced Materials, vol. 26, no. 26, pp. 4521-4526, 2014.
[40] P. V. Kumar, N. M. Bardhan, S. Tongay, J. Wu, A. M. Belcher, and J. C. Grossman, "Scalable enhancement of graphene oxide properties by thermally driven phase transformation," Nature Chemistry, vol. 6, no. 2, pp. 151-158, 2014.

[41] F. Hao, D. Fang, and Z. Xu, "Mechanical and thermal transport properties of graphene with defects," Applied Physics Letters, vol. 99, no. 4, 2011.

[42] F. Yavari, H. R. Fard, K. Pashayi et al., "Enhanced thermal conductivity in a nanostructured phase change composite due to low concentration graphene additives," Journal of Physical Chemistry C, vol. 115, no. 17, pp. 8753-8758, 2011.

[43] S. H. Song, K. H. Park, B. H. Kim et al., "Enhanced thermal conductivity of epoxy-graphene composites by using nonoxidized graphene flakes with non-covalent functionalization," Advanced Materials, vol. 25, no. 5, pp. 732-737, 2013.

[44] Y. S. Song and J. R. Youn, "Influence of dispersion states of carbon nanotubes on physical properties of epoxy nanocomposites," Carbon, vol. 43, no. 7, pp. 1378-1385, 2005.

[45] M. J. Biercuk, M. C. Llaguno, M. Radosavljevic, J. K. Hyun, A. T. Johnson, and J. E. Fischer, "Carbon nanotube composites for thermal management," Applied Physics Letters, vol. 80, no. 15, pp. 2767-2769, 2002.

[46] L. Jiang and Z. Fan, "Design of advanced porous graphene materials: from graphene nanomesh to $3 \mathrm{D}$ architectures," Nanoscale, vol. 6, no. 4, pp. 1922-1945, 2014. 

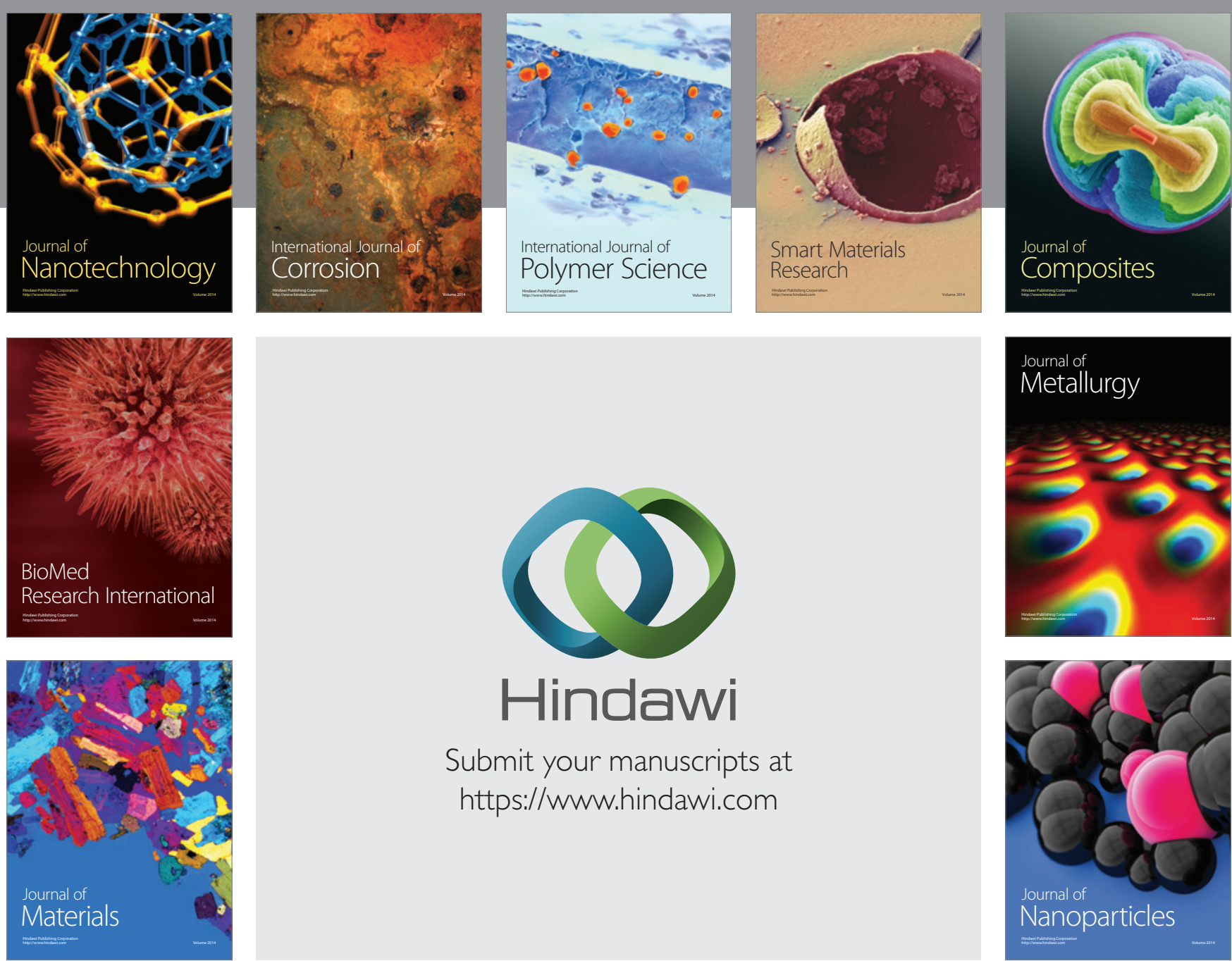

\section{Hindawi}

Submit your manuscripts at

https://www.hindawi.com

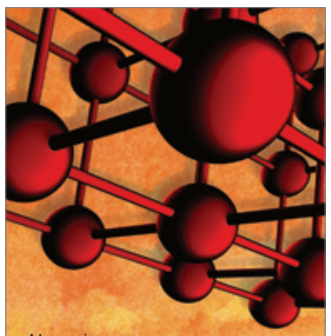

Materials Science and Engineering
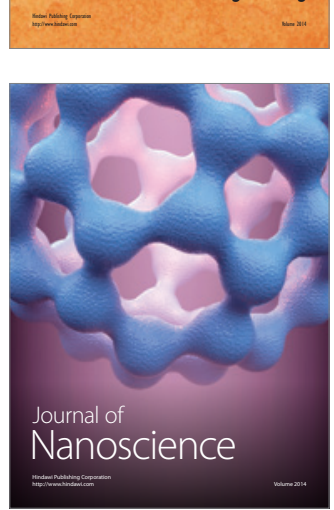
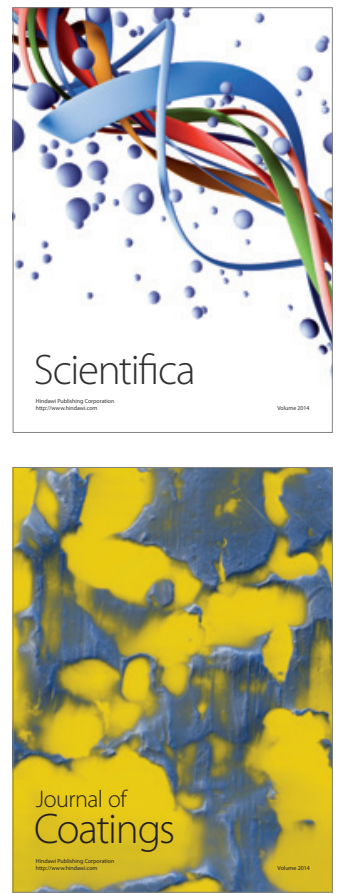
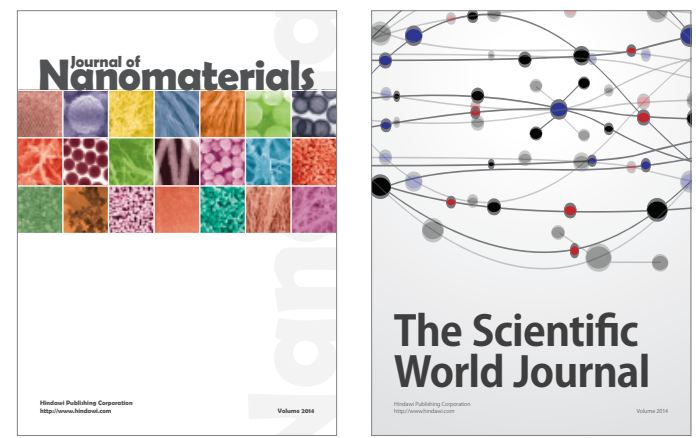

The Scientific World Journal
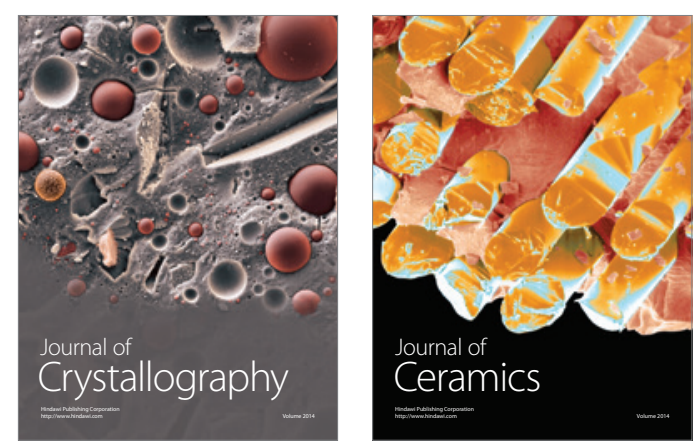
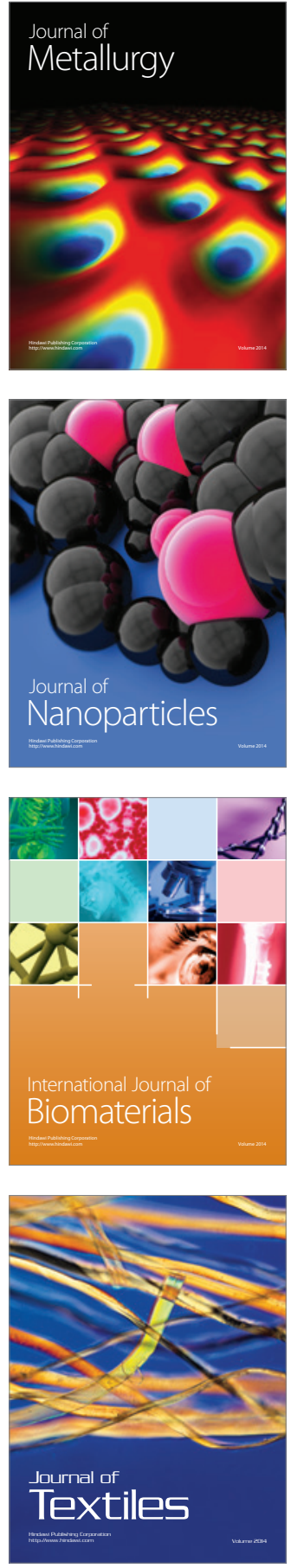